\title{
MiR-124-3p/B4GALT1 axis plays an important role in SOCS3-regulated growth and chemo-sensitivity of CML
}

Yu-xiao Liu ${ }^{1 \dagger}$, Li Wang ${ }^{2,3+}$, Wen-jia Liu ${ }^{4+}$, Hai-tao Zhang ${ }^{1}$, Jing-hui Xue ${ }^{1 *}$, Zhi-wen Zhang ${ }^{1}$ and Chun-ji Gao ${ }^{3 *}$

\begin{abstract}
Background: Abnormal expression of SOCS3 has been implicated in myeloproliferative neoplasms, but the role of SOCS3 in the pathogenesis of leukemia remains largely unknown. Here, we examined the function of SOCS3 in the growth and chemo-sensitivity of chronic myeloid leukemia (CML) and explored the involved mechanisms.

Methods: Expression levels of SOCS3 in several leukemia cell lines and bone marrow mononuclear cells (BMNCs) from CML patients were determined using quantitative real-time PCR (qPCR) and Western blotting (WB). The roles of SOCS3 in the proliferation, apoptosis, and drug resistance of CML cells were examined by clonogenic progenitor cell assay, flow cytometry, and CCK-8 assay. A detailed analysis of the underlying mechanism of SOCS3 in K562 cells was performed using the Human HT-12 v4 Expression BeadChip, which has more than 48000 gene probes including 600 microRNAs (miRNA) probes. The correlation between the mRNA expression of SOCS3 and miR-124-3p in BMNCs from 30 CML patients was tested by $\mathrm{QPCR}$ and analyzed by Pearson correlation and linear regression analysis. The potential target of miR-124-3P in CML cells was explored using the luciferase reporter assay, GPCR, and WB. The effect of SOCS3 on the miR-124-3p/B4GALT1 axis was investigated by qPCR, WB, CCK-8 assay, and tumorigenicity assays in nude mice.
\end{abstract}

Results: SOCS3 was down-regulated in CML cell lines and most of BMNCs from CML patients, and the expression level of SOCS3 was associated with the inhibition of cell proliferation and drug resistance of CML cells. Overexpression of SOCS3 in K562 cells inhibited the expression of leukemia-specific genes and promoted the expression of some miRNAs, among which miR-124-3p was the highest. SOCS3 over-expression enhanced the expression of miR-124-3p and vice versa. The mRNA expression of miR-124-3p and SOCS3 in BMNCs from 30 CML patients was positively correlated. Consistently, the tumor suppressing effects of SOCS3 were partially neutralized by the miR-124-3p inhibitor. B4GALT1 was downstream of miR-124-3p and regulated by SOCS3/miR-124-3p in vitro. Furthermore, SOCS3 over-expression could inhibit the growth and B4GALT expression of K562 cells in vivo.

Conclusions: SOCS3/miR-124-3p/B4GALT1 axis plays an important role in the pathogenesis of CML.

Keywords: SOCS3, miR-124, B4GALT1, Leukemogenesis, Chemo-sensitivity

\footnotetext{
*Correspondence: xuejh629@163.com; gaochunji@hotmail.com

${ }^{\dagger}$ Equal contributors

'Department of Neurosurgery, The First Affiliated Hospital of Chinese PLA General Hospital, 51 Fushi Road, Beijing 100048, People's Republic of China ${ }^{3}$ Department of Hematology, Chinese PLA General Hospital, 28 Fuxing Road, Beijing 100853, People's Republic of China

Full list of author information is available at the end of the article
} 


\section{Background}

Suppressor of cytokine signaling (SOCS) is a protein family of eight members (SOCS1-7 and CIS) that form a classical negative feedback system to regulate cytokine signal transduction [1]. By regulating the cytokine-driven STAT activation pathway, SOCS3 likely has an important role in development, allergic responses, and tumorigenesis [2-4]. Methylation of the SOCS3 promoter and reduced gene expression of SOCS3 have been documented in numerous tumors such as breast cancer, lung cancer, and liver cancer [5-7]. SOCS3 is highly conserved among vertebrates and has been considered to be a transcriptional regulator in the hematopoietic system. $\mathrm{SOCS}^{-/-}$mice die in utero because of fetal liver erythrocytosis, and over-expression of SOCS3 blocks fetal liver erythrocytosis, implying that SOCS3 plays a critical role in the negative regulation of hematopoiesis $[8,9]$.

Evidence suggests a potential role of SOCS3 in the pathogenesis of leukemia. For example, Capello et al revealed that inactivation of SOCS3 was frequent in $\mathrm{Ph}$ negative chronic myeloproliferative disorders (CMPD) [10]. Al-Jamal et al. reported that down-regulation of SOCS3 was involved in the resistance of CML cells to imatinib [11]. However, the exact function of SOCS3 in hematological malignancies remains unclear. A better understanding of the function and underlying molecular mechanisms of SOCS3 will contribute to the precision medicine in the field of CML.

In a previous study, we showed that SOCS3 was important for lineage commitment of hematopoietic stem cells to erythroid cells. SOCS3 knock-down increased the expression of multiple erythroid-specific genes and inhibited the expression of genes controlling lymphoid differentiation [12]. In this study, we wanted to investigate the contribution of SOCS3 in pathogenesis of $\mathrm{CML}$ and further understand the potential underlying mechanisms of SOCS3. Previous studies suggested dysregulation of microRNA (miRNA) networks had been implicated in hematological malignancies, for example miR-29a/29b dysregulation played an important role in myeloid leukemogenesis [13]. So, understanding of miRNA biology in carcinogenesis could possibly pave novel routes for anti-cancer therapy [14, 15]. Here, we found that over-expression of SOCS3 in CML cells induced a transcriptional program enriched for leukemia suppression factors, including some miRNAs. For example, miR-124-3p was obviously up-regulated by SOCS3 over-expression. In turn, alterations of miR-124$3 p$ expression levels influenced the effect of SOCS3 on CML cells. Furthermore, we confirmed that B4GALT1, a multidrug resistance gene, was the target gene of the SOCS3/miR-124-3p axis. These findings suggested the presence of a dysregulated molecular network involving SOCS3, miR-124-3p, and B4GALT1, which may provide novel insights into tumor biology and present a useful target for therapeutic interference of CML under certain circumstances.

\section{Results}

Expression of SOCS3 was dysregulated in CML cells

We measured the expression of SOCS3 by qPCR in a panel of human leukemia cell lines and primary bone marrow mononuclear cells (BMNCs) from healthy donors $(n=3)$. We found that SOCS3 expression was down-regulated in human leukemia cells, with the lowest expression levels in $\mathrm{K} 562$ cells, indicating that SOCS3 could be down-regulated in CML (Fig. 1a). We further analyzed the expression of SOCS3 in BMNCs

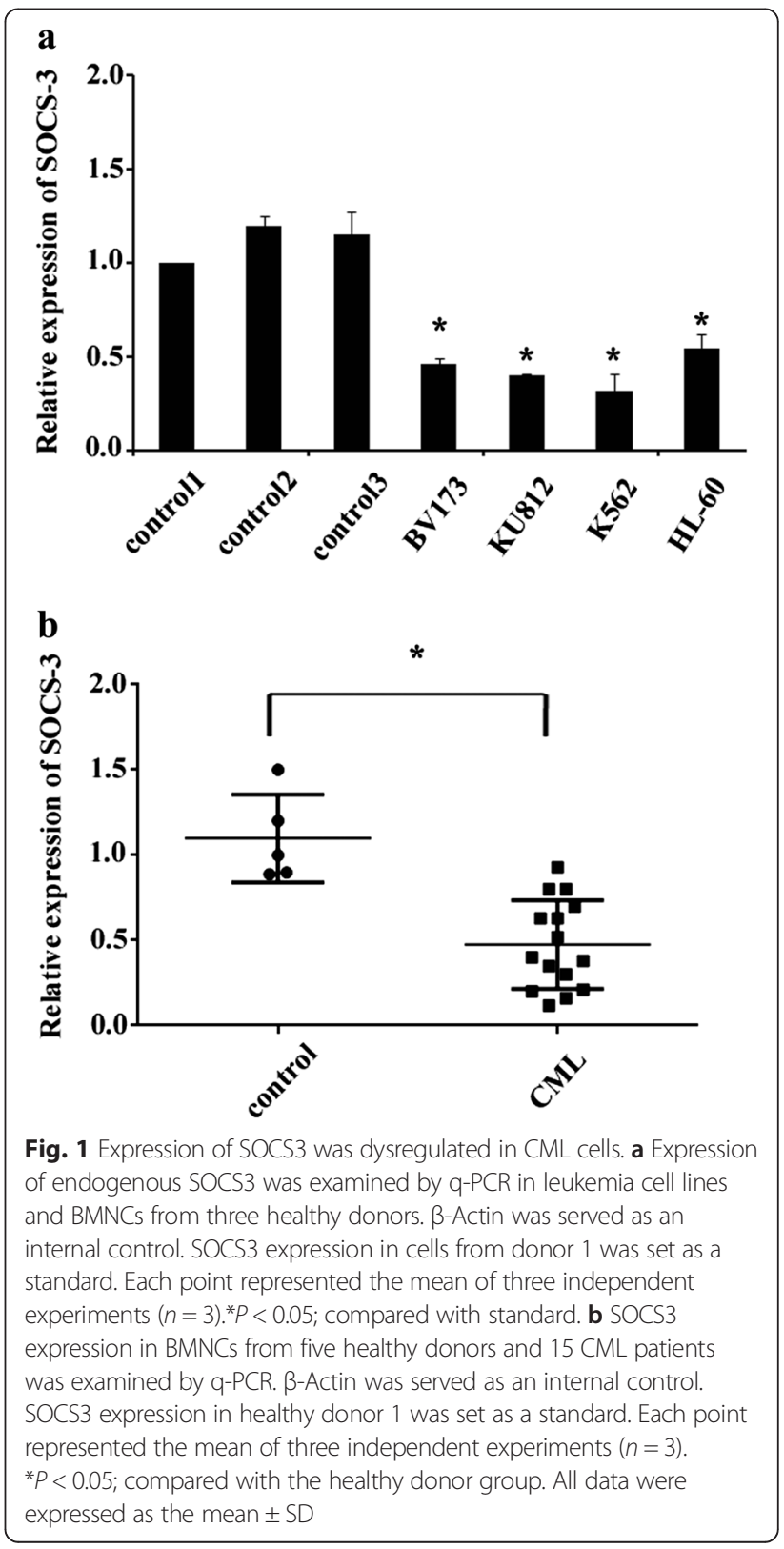


from 15 untreated CML patients. We found that compared with healthy donors $(n=5)$, SOCS3 expression was significantly down-regulated (which is below the minimal level of five healthy volunteers) in most of BMNCs from CML patients (12 of 15) (Fig. 1b). The protein expression of SOCS3 was also reduced greatly in CML cell lines and many patients (Additional file 1). All these findings suggested that expression of SOCS3 was dysregulated in CML cells.

\section{SOCS3 regulated the growth of CML cells}

To investigate the role of SOCS3 in CML cells, the expression and inference vectors of SOCS3 were stably transduced into K562 and KU812 cells using the lentiviral system. We found that SOCS3 over-expression induced a marked reduction in the number of colonies and SOCS3 knock-down led to a significant increase in the number of colonies from K562 cells by the clonogenic formation assay (Fig. 2a, b). Similar results were observed in KU812 cells (Fig. 2b). The CCK-8 assay confirmed that SOCS3 over-expression markedly inhibited the growth of K562 and KU812 cells, while SOCS3 down-regulation promoted the proliferation of these cells when compared with empty or non-targeting shRNA (shControl) groups (Fig. 2c).
SOCS3 contributed to imatinib-induced apoptosis of CML cells

We examined SOCS3 expression levels in K562 and KU812 cells prior to and after imatinib treatment. qPCR assays demonstrated a marked increase in mRNA levels of SOCS3 after imatinib treatment in two CML cell lines. Western blot assays confirmed a marked increase in the protein levels of SOCS3 after imatinib treatment (Fig. 3a). We also found that SOCS3 itself could not induce an obvious increase in apoptotic cells when K562 or KU812 cells were transduced with SOCS3 overexpression vector. However, SOCS3 over-expression could induce a marked increase in the percentage of apoptotic cells in the presence of imatinib (Fig. 3b, c).

\section{SOCS3 inhibited the expression of leukemia-specific genes and promoted a series of miRNAs}

To characterize the transcriptional changes caused by SOCS3, the gene expression profile of $\mathrm{K} 562$ was determined $48 \mathrm{~h}$ after SOCS3 over-expression or empty vector transduction. Total RNA from these cells was hybridized to an Illumina Human HT-12 v4 expression bead array. After normalization, the gene expression profile of two groups was compared using Benjamini Hochberg FDR values. Ultimately, we identified 296

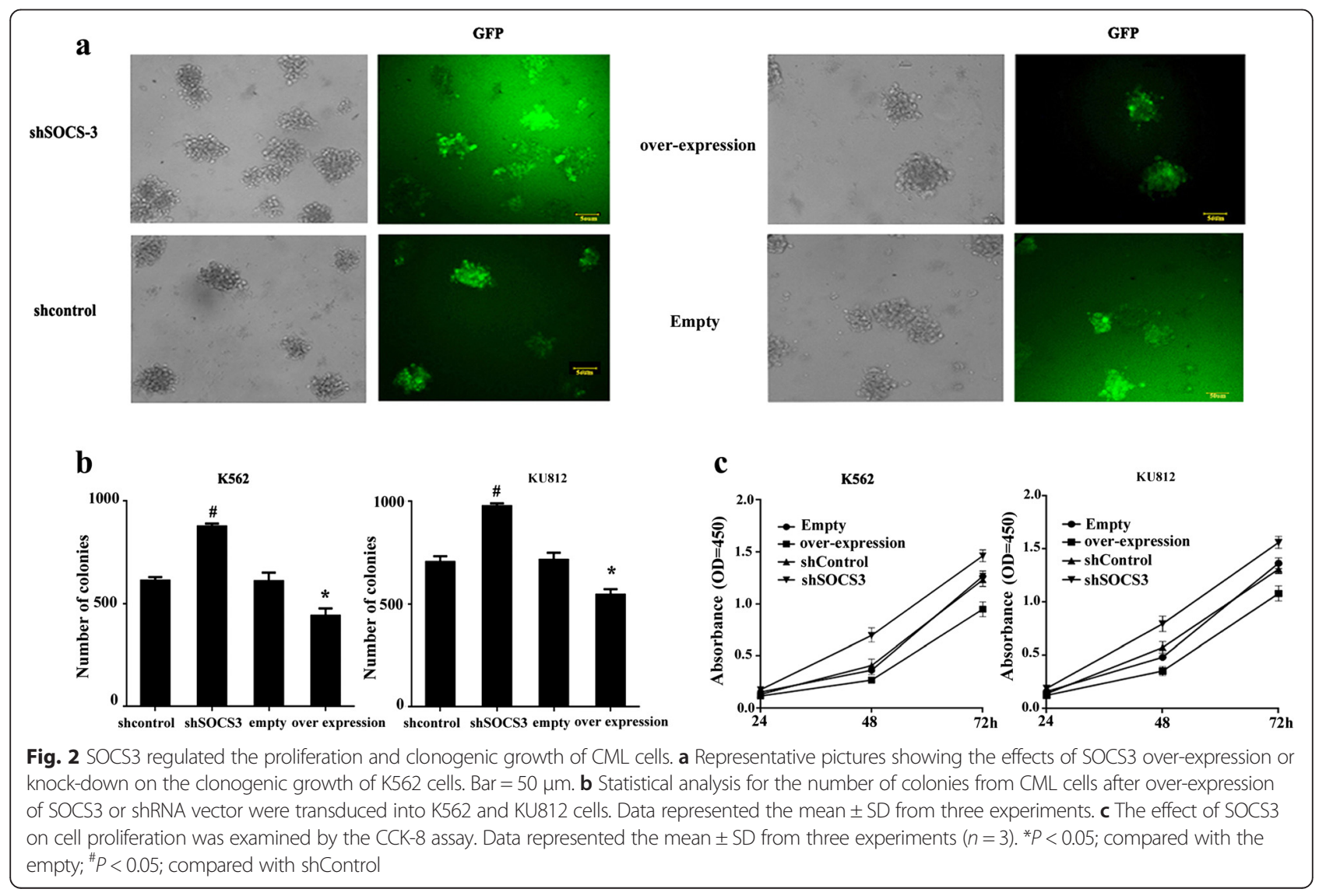




\section{a}

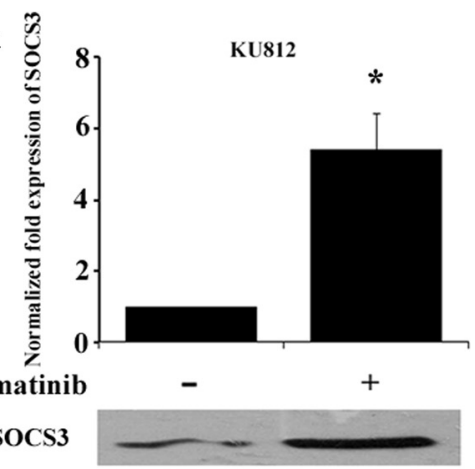

Actin
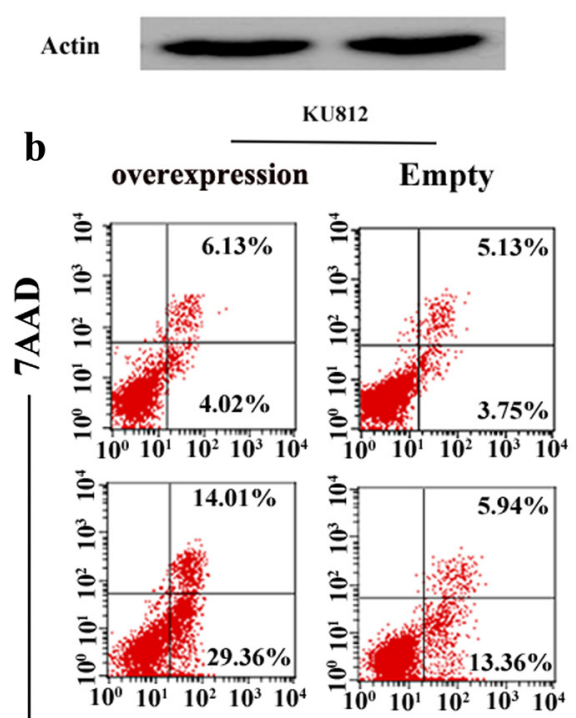

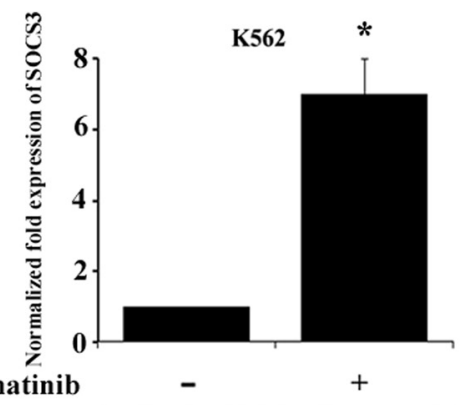

SOCS3

Actin
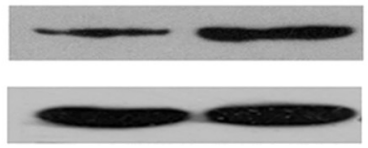

K562
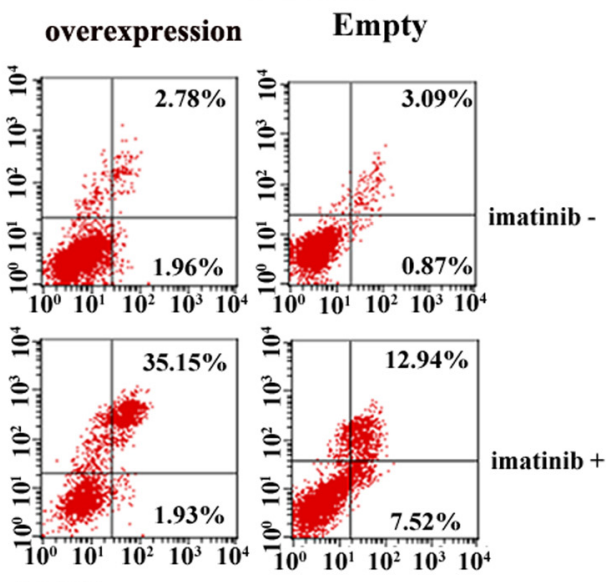

Annexin V-PE

c
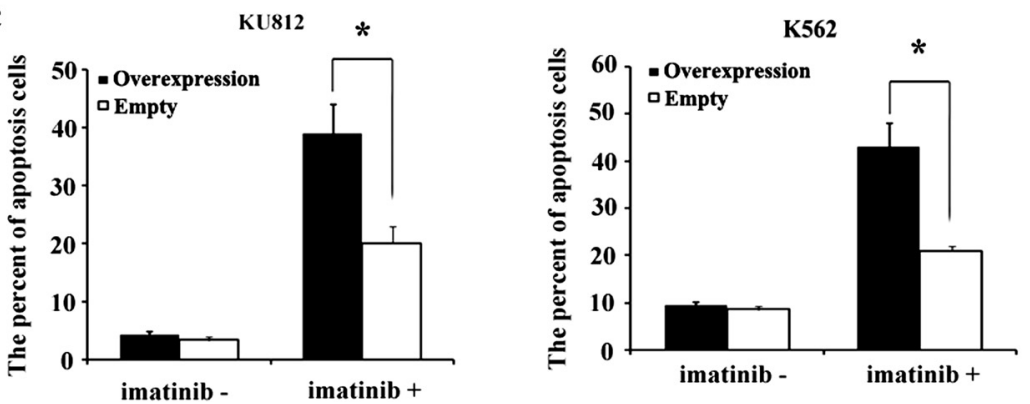

Fig. 3 SOCS3 was up-regulated by imatinib. a The expression of SOCS3 in K562 and KU812 cells was examined by qPCR and western blotting after they were treated with imatinib for $48 \mathrm{~h}$. $\beta$-Actin was served as an internal control. Each value was the mean \pm SD of three experiments $(n=3)$, ${ }^{*} P<0.05$; compared with untreated cells. b Apoptosis of K562 and KU812 cells, which were transduced with SOCS3 over-expression or empty vectors, were examined by Annexin V staining following treatment with imatinib (48 h). c Statistical analysis of apoptosis in K562 and KU812 cells after imatinib treatment. Data represented the mean \pm SD from three experiments $(n=3)$. ${ }^{*} P<0.05$; compared with cells transduced with empty vector

genes with significant differential expression in $\mathrm{K} 562$ cells that over-expressed SOCS3. Among them, 198 genes were up-regulated and 98 genes were downregulated. The pathways and genes that significantly changed were classified as follows: cancer pathways (EPAS1, CCND1, FGF13), myeloid leukemia pathways
(ZBTB16, KIT, IL13), hematopoietic cell lineage pathways (EPOR, GYPA, CD36) and so on (Fig. 4). In addition, we also found that some miRNAs were significantly affected by SOCS3 over-expression and that the expression of miR-124-3p was the highest in these miRNAs (data not shown). 


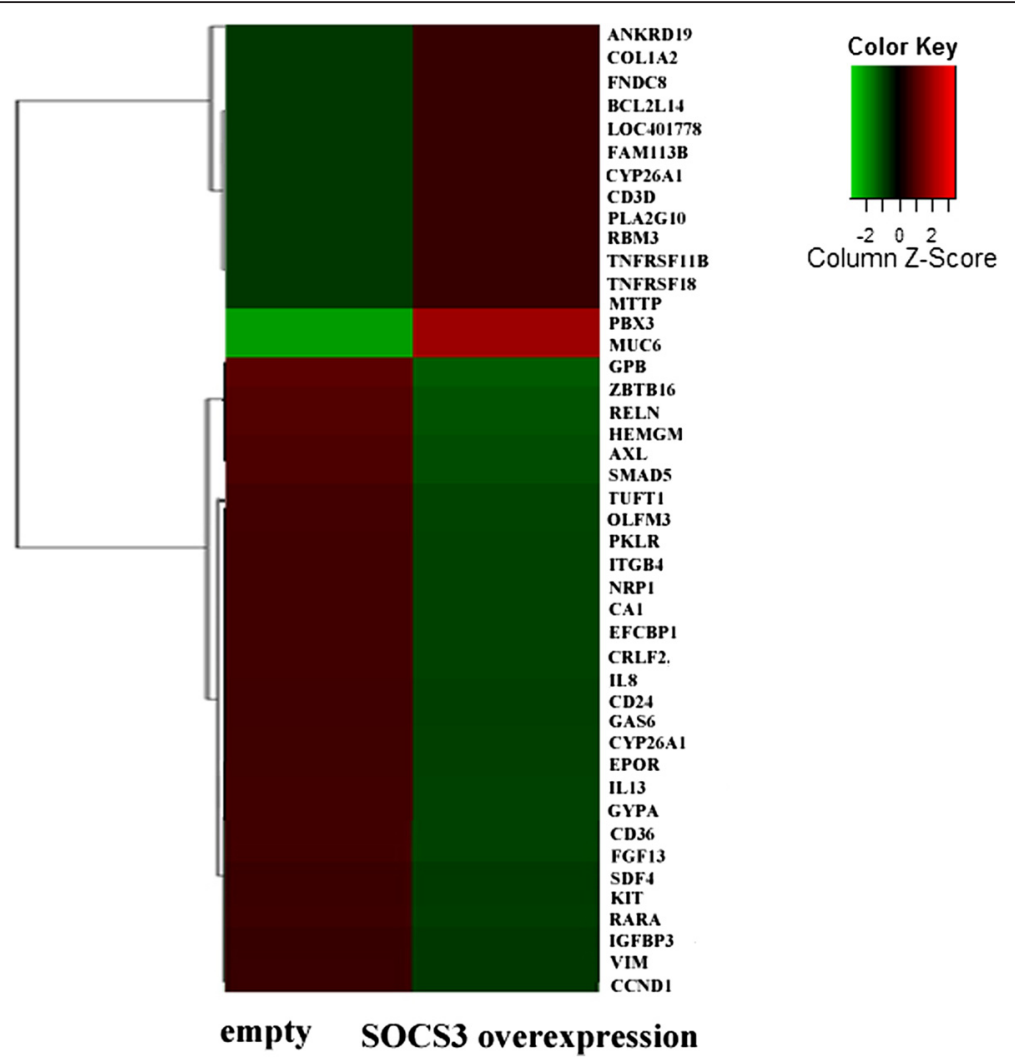

Fig. 4 The effect of SOCS3 expression on the gene expression profile of K562 cells. Heat-map showing gene expression profiles in K562 cells $48 \mathrm{~h}$ after transduction with SOCS3 over-expression or empty vectors. Each kind of sample was repeated three times by microarray $(n=3)$

Next, we investigated SOCS3-induced gene expression alteration in K562 cells using GO analysis (Additional file 2). The results confirmed that the cancer and hematopoietic cell development signaling pathways were mainly associated with responses to over-expression of SOCS3 in K562 cells.

\section{SOCS3 promoted miR-124-3p expression in CML cells}

We further confirmed the effect of SOCS3 on the expression of miR-124-3p in K562 and KU812 cell lines by q-PCR. The results showed that SOCS3 over-expression enhanced the expression of miR-124-3p, and that SOCS3 knock-down inhibited the expression of miR124-3p in both cell lines (Fig. 5 a, b). In addition, imatinib treatment resulted in a significant increase of miR-124-3p in CML cell lines, while up-regulation of miR-124-3p induced by imatinib was inhibited by SOCS3 knock-down in K562 and KU812 cells (Fig. 5c).

Next we explored the correlation between SOCS3 and miR-124-3p in BMNCs from CML patients $(n=30)$. The levels of SOCS3 and miR-124-3p were measured and normalized. As shown in Fig. 5d, when the relative expression levels of miR-124-3p were plotted against that of SOCS3 in each patient, a significant positive correlation was found (miR-124-3p vs. SOCS3: $R=0.86$, $P<0.05)$.

We next investigated whether SOCS3 regulated CML cell function by up-regulating miR-124-3p. MiR-124-3p inhibitor and negative control were transduced into K562 and KU812 cells that were stably transduced by SOCS3 over-expression vector. Growth capacity was compared with cells transduced with empty expression vector. As expected, the cell proliferation assay and clonogenic assay showed that the miR-124-3p inhibitor partially neutralized the inhibiting effects of SOCS3 (Fig. 6).

\section{B4GALT1 is a target of miR-124-3p in CML cells}

Next, we searched for potential genes regulated by miR124-3p in leukemogenesis. Using TargetScan and miRanda online search programs, we identified B4GALT1 as a potential target of miR-124-3p. A matched sequence was found at the nts 2089-2096 region of B4GALT1 mRNA 3'UTR (Fig. 7a).

To confirm that miR-124-3p targets the 3 'UTR region of B4GALT1 in CML cells, HEK293 cells were cotransduced with miR-124-3p expression or control vector along with either the full-length 3'UTR of B4GALT1(Luci-B4GALT1) or mutated Luci-B4GALT1 

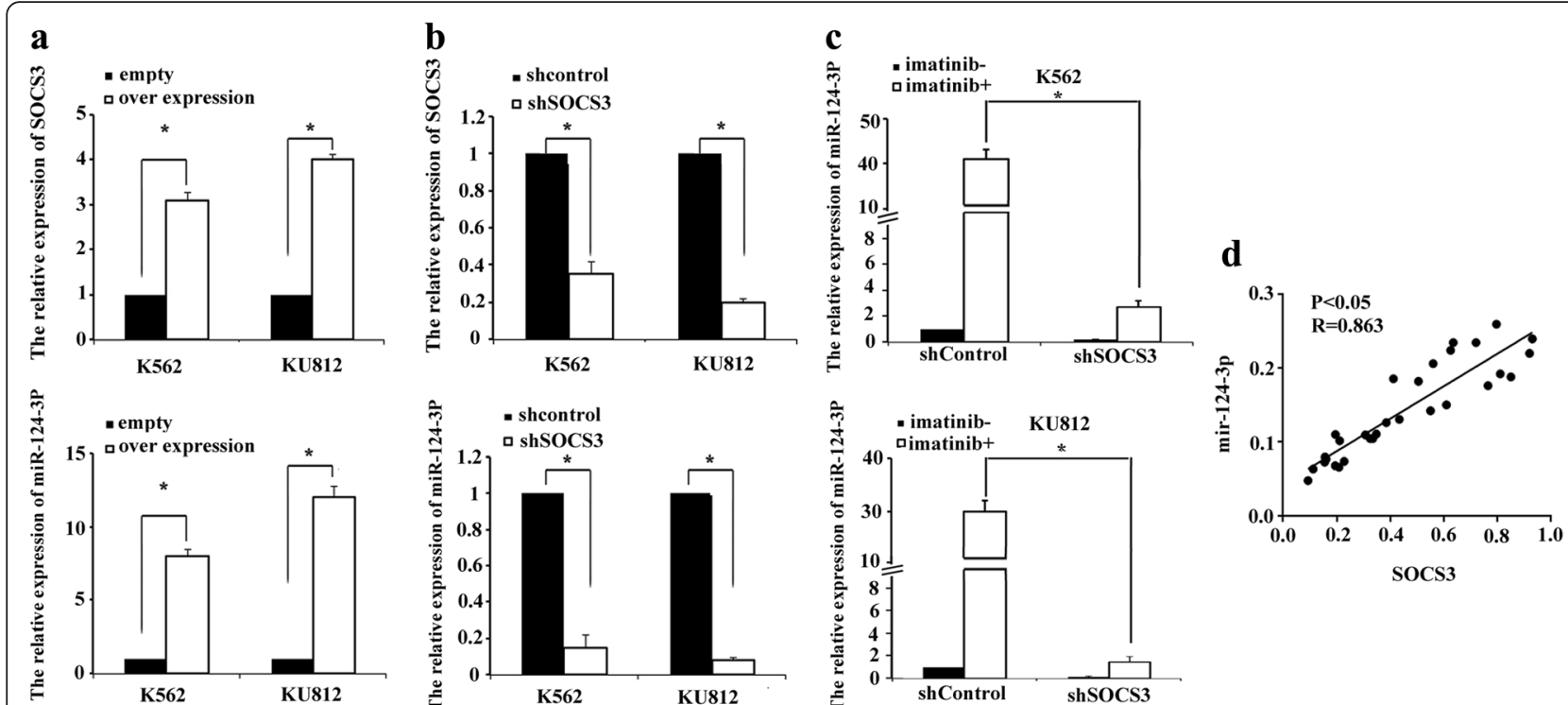

Fig. 5 SOCS3 regulated the expression of miR-124-3p in CML cells. The relative mRNA levels of SOCS3 or miR-124-3p in K562 and KU812 cells were analyzed by q-PCR after SOCS3 over-expression (a) or knock-down (b). Each value was the mean \pm SD of three experiments $(n=3)$, ${ }^{*} P<0.05$; compared with standard. c Relative mRNA levels of miR-124-3p in K562 and KU812 cells that were stably transduced with shSOCS3 or shControl vector were examined at $48 \mathrm{~h}$ following imatinib treatment. Results were normalized to untreated cells. RNU6-2 was served as an internal control. Each value was the mean \pm SD of three experiments $(n=3),{ }^{*} P<0.05$; compared with shControl. $\mathbf{d}$ Statistically significant correlation between miR-124-3p and SOCS3 expression was observed by Pearson's method. The $y$-axis or $x$-axis represented the relative mRNA levels of miR-124-3p or SOCS3 in BMNCs from 30 CML patients, which were normalized against internal control RNU6-2 or $\beta$-Actin. Each point represented the mean of three independent experiments $(n=3)$. Data are expressed as the mean \pm SD

reporter vectors bearing deletions of the $3^{\prime} \mathrm{UTR}$ target regions ( $\triangle$ Luci-B4GALT1) (Fig. 7b). We found that luciferase activity of HEK293 cells was significantly decreased after co-transduction of miR-124-3p expression vector and a 3'UTR vector containing the B4GALT1/miR-124$3 p$ target sequence (Fig. 7c).

Moreover, we over-expressed or inhibited the expression of miR-124-3p in K562 and KU812 cells and determined the endogenous expression of B4GALT1 at both the protein and mRNA level. We found that the mRNA level of B4GALT1 was not significantly affected by miR124-3p in comparison with the control in both $\mathrm{K} 562$ and KU812 cells (Fig. 7d, e). However, B4GALT1 protein was markedly reduced after transduction with miR-124$3 p$ expression vector, and vice versa (Fig. 7f). These data indicated that B4GALT1 was the target gene of miR124-3p in CML cells, and miR-124-3p suppressed B4GALT1 gene expression at the post-transcriptional level.

\section{B4GALT1 was regulated by the SOCS3/miR-124-3p axis}

The expression of B4GALT1 protein was examined in K562 cells after the expression or inference vectors of SOCS3 were stably transduced into them. The results showed that B4GALT1 expression was inhibited by SOCS3 over-expression and promoted by SOCS3 knock down in vitro (Fig. 8a). We further found that SOCS3- induced down-regulation of B4GALT1 was attenuated by the presence of the miR-124-3p inhibitor (Fig. 8b).

To investigate the impact of B4GALT1 on SOCS3induced chemo-sensitivity promotion in leukemia cells, K562 cells which stably over-expressed SOCS3 were transduced with the B4GALT1 expression vector or its negative control and then we examined apoptosis ratios of $\mathrm{K} 562$ cells in different groups after imatinib treatment. We found that ectopic expression of B4GALT1 significantly abrogated increasing chemo-sensitivity induced by SOCS3 over-expression in K562 cells (Fig. 8c).

Furthermore, K562 cells $\left(5 \times 10^{6}\right)$, which were transduced with SOCS3 over-expressing or empty vector, were inoculated into 25 -g male nude mice subcutaneously $(n=8)$. The tumor formations were observed 4 weeks after inoculation. We found the weight of tumors in the SOCS3 over-expression group was significantly lower than that of the empty vector groups (Fig. 8d). Finally, tumor tissues were collected and sectioned. Immuno-histological examination was carried out using an antibody raised against B4GALT1. Fewer B4GALT1-positive cells were observed in the tumors of SOCS3 over-expressing group (Fig. 8e).

\section{Discussion}

In this study, we explored the function and involved mechanisms of SOCS3 in the pathophysiology of CML. 

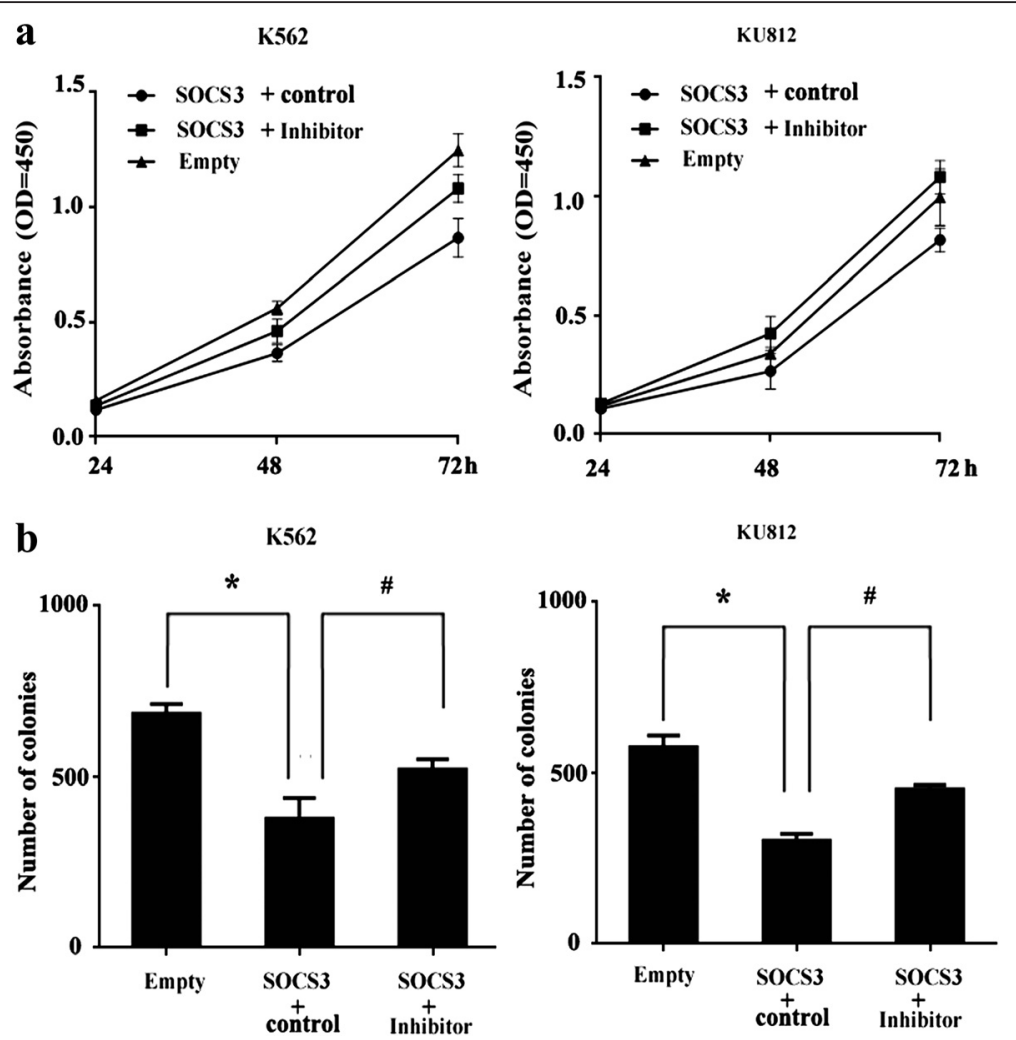

Fig. 6 miR-124-3p inhibitor partially neutralized the inhibitory effects of SOCS3. The miR-124-3p inhibitor and control were transduced into K562 and KU812 cells that were stably transduced with SOCS3 over-expression vector. The effects of miR-124-3p on cell growth (a) and colony formation (b) were determined. Data represented three independent experiments and were shown as the mean $\pm \operatorname{SD}(n=3),{ }^{*} P<0.05$; compared with empty vector or inhibitor

Firstly, we found that both mRNA and protein expression of SOCS3 were down-regulated in CML cell lines and most of samples from CML patients. Sakai et al. observed a large variation of SOCS3 expression in different patients [16]; here, we also found the significant difference of SOCS3 expression in different patients which implied SOCS3 may be used as an index for CMLprecision diagnosis in the future.

Previous studies demonstrated that the expression of SOCS3 was associated with the response of CML cells to IFN-alpha [16, 17], and down-regulation of SOCS3 was a possible reason for imatinib resistance of leukemia cells [11]. Consistently, in our study, the expression of SOCS3 in CML cells was up-regulated after imatinib treatment. Although SOCS3 exerted no remarkable effect on apoptosis, SOCS3 over-expression could enhance imatinib-induced apoptosis in CML cells. Takeuchi et al. suggested increased expression of SOCS3 in bone marrow cells may result from the action of several cytokines secreted in the bone marrow environment [17], so we speculated the bone marrow environment alteration caused by imatinib treatment may induce the upregulation of SOCS3 in CML cells here. However, the in-depth mechanism needed to be clarified.
miRNAs play important roles in tumorigenesis [1823]. We then explored whether SOCS3 regulated the growth and chemo-sensitivity of CML cells by modulating miRNA. The gene expression bead array results indicated that miR-124-3p, a tumor suppressor [24-26], was significantly affected by SOCS3. Fowler et al demonstrated that over-expression of miR-124 in GBM cells was associated with diminished tumor cell migration and invasion [27]. Moreover, Shi et al. found that miR124-3p could inhibit the proliferation of prostate cancer cells [28]. In our study, we found that miR-124-3p expression in CML cell lines was regulated by SOCS3. A significant positive correlation between miR-124-3p and SOCS3 was observed. And, the inhibitory effect of SOCS3 on CML cell proliferation was attenuated in the absence of miR-124-3p. All these data indicated that miR-124-3p play an important role in SOCS3-mediated growth inhibition.

Recent study demonstrated that B4GALT1 gene family play an important role in the resistance of human leukemia cells to therapeutic drugs. In multidrug resistance leukemia patients, highly expressed B4GALT1 regulated the hedgehog pathway, and were associated with the expression of p-glycoprotein and multidrug resistance- 


\section{a Target region}

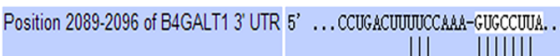

hsa-miR-124-3p. 1

b The sequence of luci- $\triangle$ B4GALT1

5 '...CCUGACUUUUCCAAAGUGCCUUAAAAGAAA....3'

d

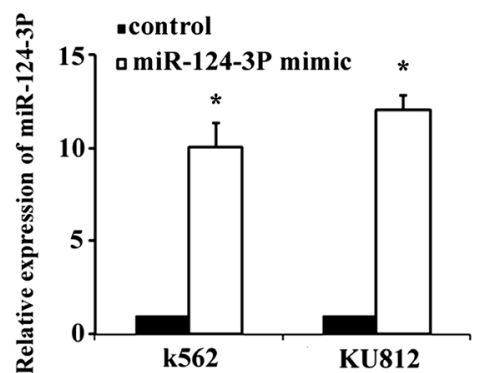

e

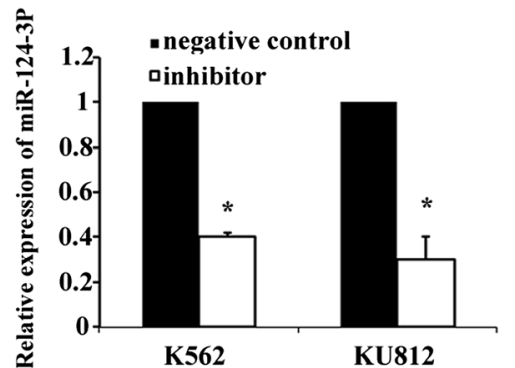

f

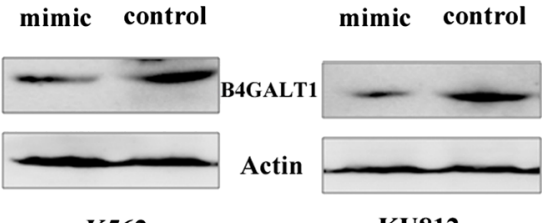

K562 c
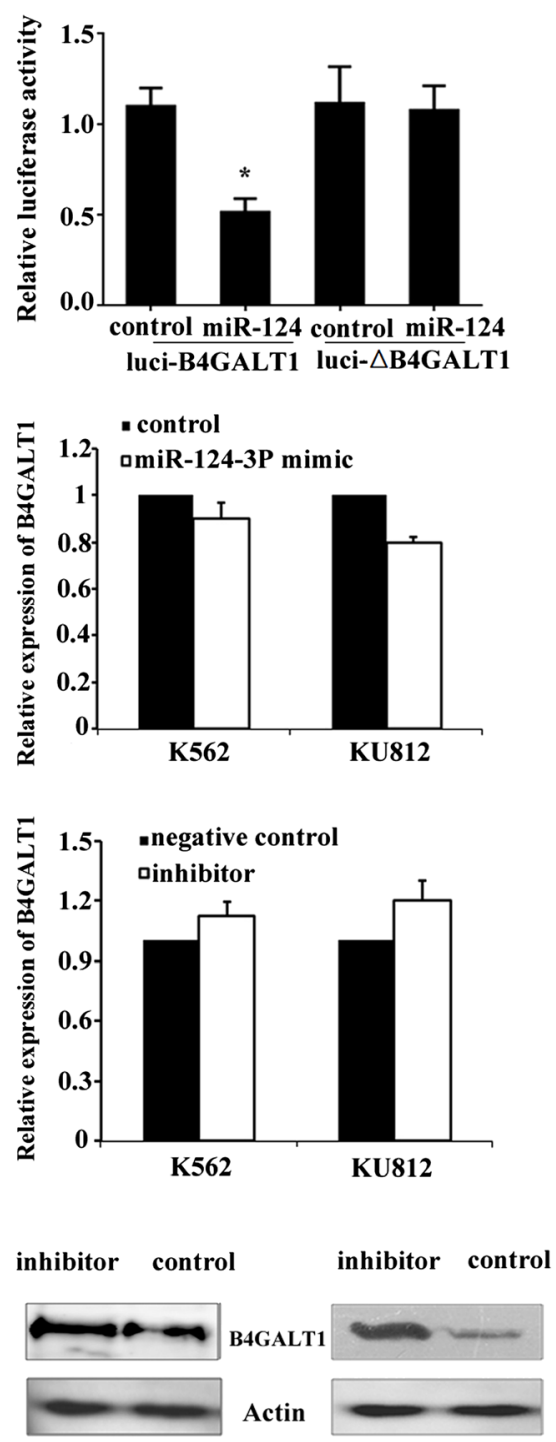

K562

KU812

Fig. 7 B4GALT1 was a target of miR-124-3p. a Bioinformatics analysis of the predicted interactions of miR-124-3p and its binding sites within the 3'UTR of B4GALT1. b Mutated sequences used in the luciferase assay. c Luciferase analysis in HEK293 cells. The expression levels of miR-124-3p or B4GALT1 in K562 and KU812 cells were analyzed by qPCR after they were transduced with miR-124-3p expression mimic (d) or inhibitor (e). U6 or $\beta$-Actin was served as an internal control. Each value was the mean \pm SD of three experiments $(n=3){ }_{,}^{*} P<0.05$; compared with control or negative control. $\mathbf{f}$ Western blot analysis of B4GALT1 expression in K562 and KU812 cells after they were transduced with miR-124-3p expression mimic or miR-124-3p inhibitor for 48 h. $\beta$-Actin was served as an internal control $(n=3)$

associated protein, resulting in the specific drug-resistant phenotypes of leukemia cell lines $[29,30]$. Here, we first demonstrated that B4GALT1 was a target gene of miR124-3p as predicted by bioinformatics, verified the conserved region in the B4GALT1 3'UTR was binding to miR-124-3p. In addition, we found that B4GALT1 protein expression was significantly down-regulated by miR-124$3 p$ over-expression in CML cells. So, we speculated that SOCS3 enhanced the chemo-sensitivity of CML cells by down-regulating B4GALT1, and that miR-124-3 was the link between them. Thus, we analyzed the effect of SOCS3 on the expression of B4GALT1 and proved that SOCS3 modulated the expression of B4GALT1 by miR-124-3p and, in turn, B4GALT1 could rescue SOCS3-induced chemosensitivity alterations in K562 cells. Finally, tumorigenicity assays in nude mice confirmed that over-expression of SOCS3 inhibited the proliferation of K562 cells and downregulated the expression of B4GALT1 in vivo. However, the in vivo function of SOCS-3 after imatinib treatment needs to be investigated in the further study.

Our results showed SOCS-3 regulated miR-124-3p/ B4GALT1 pathway played an important role in the 


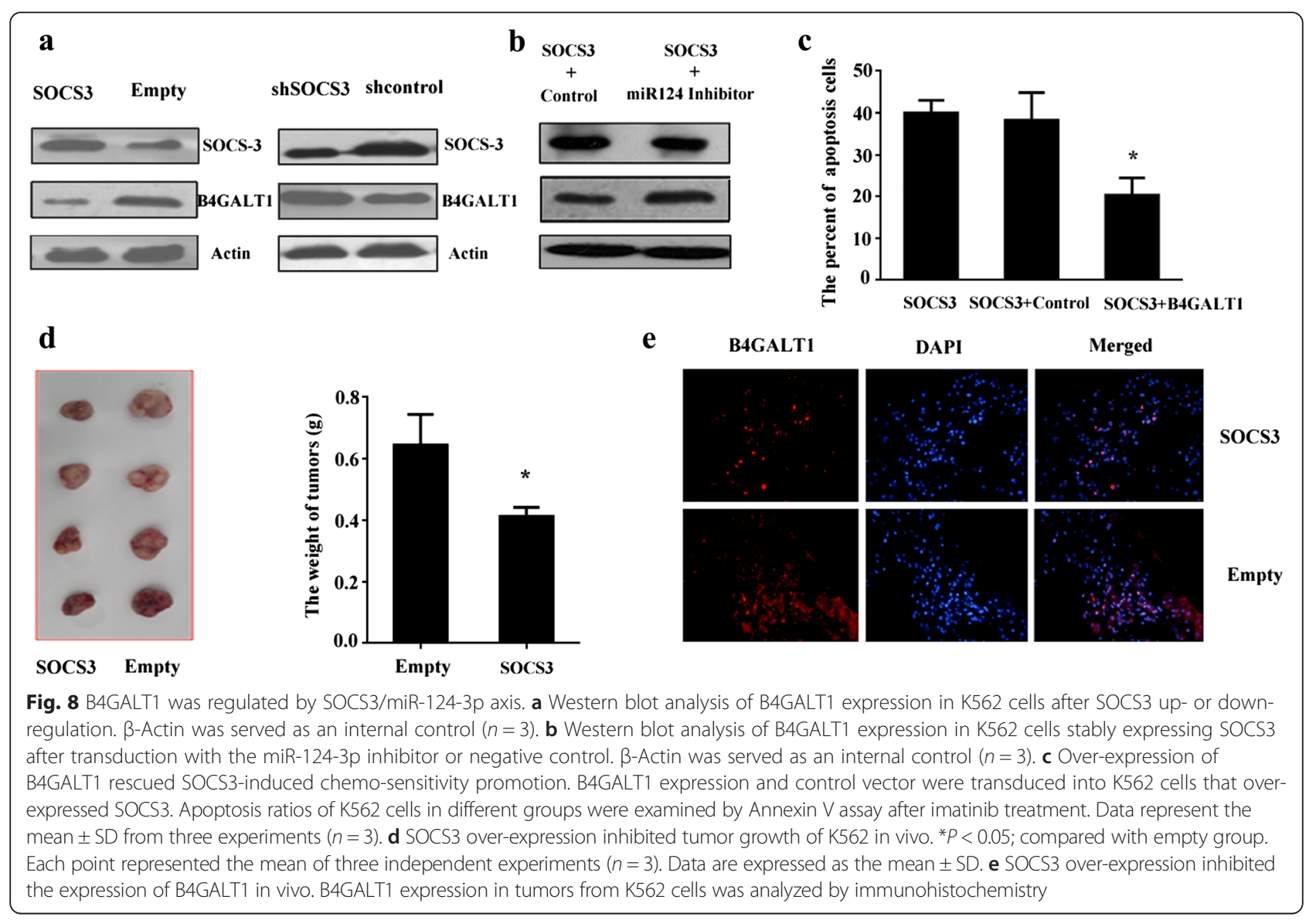

pathogenesis of CML. However, imatinib treatment still induced miR-124-3p increase in the absence of SOCS3 and SOCS3 could inhibit colony formation, regardless of the presence of miR-124-3p inhibitor, which implied other signal pathways may be involved. For example, previous studies demonstrated JAK/STAT pathway and cytokine signal pathways were involved in SOCS3mediated effects in CML cells $[16,17]$.

Resistance to targeted drugs remains a challenge for CML therapy. Accurate biomarkers are of great importance to antitumor therapeutics [31]. In this study, there was an obvious correlation between SOCS3 expression and the sensitivity of CML cell lines to imatinib. Thus, SOCS3 may be used as a novel biomarker predicting the response to targeted drugs and it was of great value to further elucidate the role and mechanism underlying SOCS3 expression in CML cells. However, we did not explore the role of SOCS3 in primary cells from CML patients who are resistant to imatinib, and we acknowledge this is a limitation of this study.

\section{Conclusions}

In summary, our work revealed that an interesting signal pathway initiated by SOCS3 was involved in CML development. Down-regulated SOCS3 in CML cells was associated with low level of miR-124-3p, then could not exert enough repressive effect on B4GALT1, resulting in the proliferation of CML cells and targeted drugs resistance. In conclusion, SOCS3/miR-124-3p/B4GALT1 signaling pathway plays an important role in the pathophysiology of CML. SOCS3 may be used as an index for CML diagnosis, and a novel biomarker predicting the response of CML to targeted drugs, in clinical settings.

\section{Methods}

\section{Patient samples}

BMNCs were obtained from patients with confirmed diagnose of CML and from healthy donors with informed consent. BMNCs were enriched by Ficoll gradient centrifugation. The study was performed with the approval of the Ethics Committee of Chinese PLA General Hospital, Beijing, China.

\section{Cell culture}

K562, KU812, HL-60, and BV173 cells were cultured in RPMI-1640. HEK293 cells were cultured in DMEM. These mediums contained $10 \%(\mathrm{v} / \mathrm{v})$ fetal bovine serum (Gibco, Life technologies, USA) and $100 \mathrm{mg} / \mathrm{mL}$ 
penicillin/streptomycin. For imatinib treatment, cells were treated with $1 \mu \mathrm{mol} / \mathrm{L}$ imatinib for $48 \mathrm{~h}$.

\section{Lentiviral plasmid construct and transduction}

Lentiviral expression and interference vectors targeting human SOCS3 were constructed as described previously [12]. Empty expression vector (empty) or non-targeting siRNA (shControl) were used as controls of expression and interference vectors, respectively. Lentiviral particles were produced and cells were transduced. After transduction $(48 \mathrm{~h})$, positive cells were sorted by fluorescenceactivated cell sorting (FACS), according to the expression of green fluorescent protein (GFP).

\section{Quantitative RT-PCR}

Total RNA isolation, reverse transcription, and the quantification of target gene expression were performed as previously described [12]; $\beta$-Actin was used as an internal control. miR-124-3p expression levels were quantified using U6 as the internal control (GenePharm). The foldchange in expression was calculated using the following primer pairs for the amplification of target mRNAs: SOCS3 forward primer 5'ATCCTGGTGACAT GCTCCTC'3 and reverse primer 5'CAAATGTTGCTTCCCCCTTA'3; $\beta$ Actin forward primer $5^{\prime}$ GATCCACATCTGCTGGAA GG'3 and reverse primer 5'AAGTGTGACGTT GAC ATCCG'3; B4GALT1 forward primer 5'AACCATGT GACTGAGTGC CC'3 and reverse primer 5'TCAG TGTGTTGTGCCAAAGC'3; Micro124-3p forward primer 5'TAAGGCACGCGGTGAATGCC'3 and reverse primer 5'GATTGAATCGA GCACCAG TTAC’3; U6 forward primer 5'CGCTTCGGCAG CAC ATATACTA’3 . Unified reverse primer 5‘GATTGAATCGA GCACCAG TTAC’3.

\section{Western blot analysis}

Cells were lysed directly in lysis buffer to collect whole cell extracts. Protein samples were separated on polyacrylamide gels, transferred onto nitrocellulose membrane by iblot (Invitrogen), detected using horseradish peroxidase-conjugated secondary antibodies, and exposed to BioMax film (Kodak) following chemiluminescence (Santa Cruz, CA, USA). The following primary antibodies were used: SOCS3, Actin, and B4GALT1 (Santa Cruz, CA, USA).

\section{Cell proliferation assay}

Cell proliferation was determined using CCK-8 (Dojindo, Japan) method. K562 cells (3000 cells/well) were plated in 96-well plates. At different time points, CCK-8 reagents were added to each well and further incubated at $37{ }^{\circ} \mathrm{C}$ for $2 \mathrm{~h}$. The number of viable cells was assessed by measurement of absorbance at $450 \mathrm{~nm}$ using a Multiskan (Thermo Scientific, Asheville, NC, USA).

\section{Clonogenic progenitor cell assay}

Cells were seeded in a 6-well plate with methylcellulose medium (MethoCult H4435, STEMCELL Technologies, Canada) according to the manufacturer's instructions. After 1 week of cultivation, colonies were counted.

\section{Apoptosis assay}

The apoptosis assays were performed using the Annexin V-PE kit (BioLegend, San Diego, CA, USA) according to the manufacturer's protocol. The stained cells were immediately analyzed on a FACScalibur flow cytometer (Becton Dickinson). The data were expressed as the percentage of apoptosis cells.

\section{Whole-genome expression analysis}

Total RNA was extracted from K562 cells transduced with over-expression SOCS3 or empty vector $\left(5 \times 10^{6}\right.$ cells) using Trizol reagent (Invitrogen Life Technologies, Paisley, UK). Genome expression analysis was performed by Illumina Human HT-12 v4 BeadChip (Illumina, San Diego, CA, USA) at the Beijing Qian zhao xing ye Biological Technology Co., Ltd. (Beijing, China).

\section{miR-124-3p mimic and inhibitor}

The hsa-miR-124-3p mimic or control sequence, and hsa-miR-124-3p inhibitor and hsa-miR-124-3p inhibitor negative control were all purchased from GenePharma (Shanghai, China).

\section{Luciferase assays}

The human pre-miR-124 sequence was amplified and cloned into pcDNA3.1 constructs (Invitrogen) to generate the pcDNA3.1-miR-124 expression vector. The fulllength 3'UTR of B4GALT1 was amplified using cDNA from $\mathrm{K} 562$ cells and double-digested with XbaI/EcoRI and cloned downstream of the firefly luciferase coding region sites of a modified pGL3-control plasmid named Luci-B4GALT1. We also constructed mutated LuciB4GALT1 reporter vectors bearing deletions of the UTR target regions and named them Luci- $\triangle$ B4GALT1. The vectors were co-transduced with control or pcDNA3.1miR-124 expression vectors into K562 cells. Lysates were prepared $48 \mathrm{~h}$ after transduction. Luciferase activity was measured using a dual-luciferase reporter assay system (Promega). All experiments were performed in triplicate at least three independent times.

B4GALT1 expression plasmid construction and transduction The full-length of the human B4GALT1 sequence was amplified and cloned into the pcDNA3.1 vector to generate the B4GALT1 expression vector. K562 cells were transduced with the B4GALT1 expression vector in 24well plates using Lipofectamine 2000 (Invitrogen) according to the manufacturer's protocol. 


\section{Tumorigenicity assays in nude mice}

A total of $5 \times 10^{6} \mathrm{~K} 562$ cells that were stably transduced with SOCS3 over-expression or empty vectors were injected into male nude mice subcutaneously $(n=8)$. Mice were sacrificed at 28 days post-inoculation, and the tumors were excised and their weight was measured and photographed. Tumors from different groups were removed and fixed with formaldehyde, embedded in paraffin wax, and sectioned. The sections were cleared through xylene, graded ethanol, water and incubated with anti-B4GALT1 antibodies (Santa Cruz) at $4{ }^{\circ} \mathrm{C}$ overnight, stained with DAPI, washed with PBS three times, and observed by fluorescence microscopy.

\section{Statistical analysis}

All data were expressed as the mean \pm standard deviation (SD). The differences between two groups were assessed by Student's $t$ test. Pearson's correlation coefficient was calculated to analyze the correlation. $P<0.05$ was considered to be statistically significant.

\section{Additional files}

Additional file 1: Figure S1. Expression of SOCS3 protein in CML cells. SOCS3 expression in CML cell lines and BMNCs from CML patients was analyzed by Western blotting. $\beta$-Actin was served as an internal control $(n=3)$. (TIF $69 \mathrm{~kb})$

Additional file 2: Figure S2. GO analysis of SOCS3-induced gene expression alteration in $\mathrm{K} 562$ cells. Branches of the $\mathrm{GO}$ hierarchical tree with significantly enriched $\mathrm{GO}$ terms were indicated in red boxes. Insignificant GO terms within the hierarchical tree are shown as white boxes. (TIF $203 \mathrm{~kb}$ )

\section{Abbreviations}

3'-UTR, 3'-untranslated region; BMNCs, bone marrow mononuclear cells; CML, chronic myeloid leukemia; CMPD, chronic myeloproliferative disorders; FACS, fluorescence - activated cell sorting; GFP, green fluorescent protein; miR-124$3 p$, microRNA-124-3p; miRNAs, microRNAs; qPCR, quantitative real-time PCR; siRNA, small interfering RNA; SOCS3, suppressor of cytokine signaling 3; WB, Western blotting

\section{Acknowledgements}

Not applicable.

\section{Funding}

This study was supported by grant from the National Natural Sciences foundation of China (Grant number: 81100329 and 81472372) and the International Science and Technology Cooperation Program of China (2011DFA30550). The funders had no role in study design, data collection and analysis, decision to publish, or preparation of the manuscript.

\section{Availability of data and materials}

The dataset supporting the conclusions of this article are included within the article and its additional file.

\section{Authors' contributions}

$Y X L, Z Z H, J H X$, and $C J G$ participated in designing the research. $Y X L$ and $L W$ performed the research and data analysis. WJL and HTZ performed the luciferase reporter assay experiments. LW and CJG contributed the patient samples and participated in their characterization. WJL performed tumorigenicity assays. YXL wrote the paper. All authors read and approved the final manuscript.

\section{Competing interests}

The authors declare that they have no competing interests.

\section{Consent for publication}

Not applicable.

\section{Ethics approval and consent to participate}

RNA from patients was isolated from the peripheral blood of CML patients after written informed consent to research studies, and the study was approved by the Ethics Committee of Chinese PLA General Hospital. Al animal experiments were conducted in agreement with the Guide for the Care and Use of Laboratory Animals and were approved by the Ethics Committee of the Chinese PLA General Hospital.

\section{Author details}

'Department of Neurosurgery, The First Affiliated Hospital of Chinese PLA General Hospital, 51 Fushi Road, Beijing 100048, People's Republic of China. ${ }^{2}$ Department of Hematology, Chinese PLA General Hospital, Laoshan Branch, No. 401, Qingdao 266100, People's Republic of China. ${ }^{3}$ Department of Hematology, Chinese PLA General Hospital, 28 Fuxing Road, Beijing 100853, People's Republic of China. ${ }^{4}$ Genetic Laboratory of Development and Diseases, Beijing Institute of Biotechnology, Beijing 100071, People's Republic of China.

Received: 28 July 2016 Accepted: 5 August 2016

Published online: 12 August 2016

\section{References}

1. Kershaw NJ, Murphy JM, Liau NP, Varghese LN, Laktyushin A, Whitlock EL, Lucet IS, Nicola NA, Babon JJ. SOCS3 binds specific receptor-JAK complexes to control cytokine signaling by direct kinase inhibition. Nat Struct Mol Biol. 2013;20(4):469-76.

2. Culig Z. Suppressors of cytokine signalling-3 and -1 in human carcinogenesis. Front Biosci (Schol Ed). 2013;5:277-83.

3. Ramana CV, Kumar A, Enelow R. Stat1-independent induction of SOCS-3 by interferon-gamma is mediated by sustained activation of Stat3 in mouse embryonic fibroblasts. Biochem Biophys Res Commun. 2005;327(3):727-33.

4. Roberts AW, Robb L, Rakar S, Hartley L, Cluse L, Nicola NA, Metcalf D, Hilton DJ, Alexander WS. Placental defects and embryonic lethality in mice lacking suppressor of cytokine signaling 3. Proc Natl Acad Sci U S A. 2001;98(16): 9324-9.

5. Lee JH, Kim C, Sethi G, Ahn KS. Brassinin inhibits STAT3 signaling pathway through modulation of PIAS-3 and SOCS-3 expression and sensitizes human lung cancer xenograft in nude mice to paclitaxel. Oncotarget. 2015;6(8): 6386-405.

6. Li G, XU J, Wang Z, Yuan Y, Li Y, Cai S, He Y. Low expression of SOCS-1 and SOCS-3 is a poor prognostic indicator for gastric cancer patients. J Cancer Res Clin Oncol. 2015;141(3):443-52.

7. Niwa Y, Kanda H, Shikauchi Y, Saiura A, Matsubara K, Kitagawa T, Yamamoto J, Kubo T, Yoshikawa H. Methylation silencing of SOCS-3 promotes cell growth and migration by enhancing JAK/STAT and FAK signalings in human hepatocellular carcinoma. Oncogene. 2005;24(42):6406-17.

8. Marine JC, McKay C, Wang D, Topham DJ, Parganas E, Nakajima H, Pendeville H, Yasukawa H, Sasaki A, Yoshimura A, Ihle JN. SOCS3 is essential in the regulation of fetal liver erythropoiesis. Cell. 1999;98(5):617-27.

9. Sarna MK, Ingley E, Busfield SJ, Cull VS, Lepere W, McCarthy DJ, Wright MJ, Palmer GA, Chappell D, Sayer MS, Alexander WS, Hilton DJ, Starr R, Watowich SS, Bittorf T, Klinken SP, Tilbrook PA. Differential regulation of SOCS genes in normal and transformed erythroid cells. Oncogene. 2003; 22(21):3221-30

10. Capello D, Deambrogi C, Rossi D, Lischetti T, Piranda D, Cerri M, Spina V, Rasi S, Gaidano G, Lunghi M. Epigenetic inactivation of suppressors of cytokine signalling in Philadelphia-negative chronic myeloproliferative disorders. Br J Haematol. 2008;141(4):504-11.

11. Al-Jamal HA, Jusoh SA, Yong AC, Asan JM, Hassan R, Johan MF. Silencing of suppressor of cytokine signaling-3 due to methylation results in phosphorylation of STAT3 in imatinib resistant BCR-ABL positive chronic myeloid leukemia cells. Asian Pac J Cancer Prev. 2014;15(11):4555-61.

12. Liu YX, Dong X, Gong F, Su N, Li SB, Zhang HT, Liu JL, Xue JH, Ji SP, Zhang ZW. Promotion of erythropoietic differentiation in hematopoietic stem cells by SOCS3 knock-down. PLoS One. 2015;10(8):e0135259. 
13. Xu L, Xu Y, Jing Z, Wang X, Zha X, Zeng C, Chen S, Yang L, Luo G, Li B, Li Y. Altered expression pattern of miR-29a, miR-29b and the target genes in myeloid leukemia. Exp Hematol Oncol. 2014;3:17.

14. Dzikiewicz-Krawczyk A. MicroRNA-binding site polymorphisms in hematological malignancies. J Hematol Oncol. 2014;7:83.

15. Naidu S, Magee P, Garofalo M. MiRNA-based therapeutic intervention of cancer. J Hematol Oncol. 2015;8:68.

16. Sakai I, Takeuchi K, Yamauchi H, Narumi H, Fujita S. Constitutive expression of SOCS3 confers resistance to IFN-alpha in chronic myelogenous leukemia cells. Blood. 2002;100(8):2926-31.

17. Takeuchi K, Sakai I, Narumi H, Yasukawa M, Kojima K, Minamoto Y, Fujisaki T, Tanimoto K, Hara M, Numata A, Gondo H, Takahashi M, Fujii N, Masuda K, Fujita S. Expression of SOCS3 mRNA in bone marrow cells from CML patients associated with cytogenetic response to IFN-alpha. Leuk Res. 2005:29(2):173-8.

18. An L, Liu Y, Wu A, Guan Y. microRNA-124 inhibits migration and invasion by down-regulating ROCK1 in glioma. PLoS One. 2013;8(7):e69478.

19. Asghari F, Haghnavaz N, Baradaran B, Hemmatzadeh M, Kazemi T. Tumor suppressor microRNAs: targeted molecules and signaling pathways in breast cancer. Biomed Pharmacother. 2016;81:305-17.

20. Lee YS, Dutta A. MicroRNAs in cancer. Annu Rev Pathol. 2009;4:199-227.

21. Li Y, Wang Y, Yu L, Sun C, Cheng D, Yu S, Wang Q, Yan Y, Kang C, Jin S, An T, Shi C, Xu J, Wei C, Liu J, Sun J, Wen Y, Zhao S, Kong Y. miR-146b-5p inhibits glioma migration and invasion by targeting MMP16. Cancer Lett. 2013;339(2):260-9.

22. Liu $\mathrm{M}$, Chen $\mathrm{H}$. The role of microRNAs in colorectal cancer. J Genet Genomics. 2010;37(6):347-58.

23. Pierson J, Hostager B, Fan R, Vibhakar R. Regulation of cyclin dependent kinase 6 by microRNA 124 in medulloblastoma. J Neuro-Oncol. 2008:90(1):1-7.

24. Huang TC, Chang HY, Chen CY, Wu PY, Lee H, Liao YF, Hsu WM, Huang HC, Juan HF. Silencing of miR-124 induces neuroblastoma SK-N-SH cell differentiation, cell cycle arrest and apoptosis through promoting AHR. FEBS Lett. 2011;585(22):3582-6.

25. Silber J, Lim DA, Petritsch C, Persson Al, Maunakea AK, Yu M, Vandenberg SR, Ginzinger DG, James CD, Costello JF, Bergers G, Weiss WA, Alvarez-Buylla A, Hodgson JG. miR-124 and miR-137 inhibit proliferation of glioblastoma multiforme cells and induce differentiation of brain tumor stem cells. BMC Med. 2008:6:14

26. Zhang YH, Wang QQ, Li H, Ye T, Gao F, Liu YC. miR-124 radiosensitizes human esophageal cancer cell TE-1 by targeting CDK4. Genet Mol Res. 2016;15(2). doi:10.4238/gmr.15027893.

27. Fowler A, Thomson D, Giles K, Maleki S, Mreich E, Wheeler H, Leedman $\mathrm{P}$, Biggs M, Cook R, Little N, Robinson B, McDonald K. miR-124a is frequently down-regulated in glioblastoma and is involved in migration and invasion. Eur J Cancer. 2011:47(6):953-63.

28. Shi XB, Xue L, Ma AH, Tepper CG, Gandour-Edwards R, Kung HJ, deVere White RW. Tumor suppressive miR-124 targets androgen receptor and inhibits proliferation of prostate cancer cells. Oncogene. 2013;32(35):4130-8.

29. Zhou H, Ma H, Wei W, Ji D, Song X, Sun J, Zhang J, Jia L. B4GALT family mediates the multidrug resistance of human leukemia cells by regulating the hedgehog pathway and the expression of p-glycoprotein and multidrug resistance-associated protein 1. Cell Death Dis. 2013;4:e654.

30. Zhou H, Zhang Z, Liu C, Jin C, Zhang J, Miao X, Jia L. B4GALT1 gene knockdown inhibits the hedgehog pathway and reverses multidrug resistance in the human leukemia K562/adriamycin-resistant cell line. IUBMB Life. 2012;64(11):889-900.

31. Smith AD, Roda D, Yap TA. Strategies for modern biomarker and drug development in oncology. J Hematol Oncol. 2014;7:70.

\section{Submit your next manuscript to BioMed Central and we will help you at every step:}

- We accept pre-submission inquiries

- Our selector tool helps you to find the most relevant journal

- We provide round the clock customer support

- Convenient online submission

- Thorough peer review

- Inclusion in PubMed and all major indexing services

- Maximum visibility for your research

Submit your manuscript at www.biomedcentral.com/submit
Biomed Central 